\title{
Self-Organized Criticality in Economic Fluctuations: The Age of Maturity
}

\author{
Claudio Tebaldi * \\ Department of Finance, IGIER and Baffi Carefin, Università Bocconi, Milan, Italy
}

Self-Organized Criticality (SOC) has been proposed as a paradigm that may rationalize the emergence of macrofinancial fluctuations. The wave of innovative thinking sparked by this proposal continues to produce interesting contributions in many areas of economics, ranging from macroeconomics to finance. In this review, we propose a guided tour to these achievements, highlighting that analysis of SOC equilibria is a promising avenue to establish a nexus between i) a statistical equilibrium characterized by the spontaneous emergence of dynamic critical fluctuations and ii) a strategic equilibrium concept modeling a large number of interacting players. The critical state is the stable outcome arising from a trade-off between cooperation and competition.

Keywords: self-organized critical behavior, macroeconomics and financial markets, econometrics, critical phenomena, renorm group equation

\section{OPEN ACCESS}

Edited by:

Subhrangshu Sekhar Manna, S. N. Bose National Centre for Basic Sciences, India

Reviewed by: Attilio L. Stella,

University of Padua, Italy Bikas K. Chakrabarti, Saha Institute of Nuclear Physics (SINP), India

${ }^{*}$ Correspondence: Claudio Tebaldi claudio.tebaldi@unibocconi.it

Specialty section: This article was submitted to Interdisciplinary Physics, a section of the journal

Frontiers in Physics

Received: 12 October 2020

Accepted: 21 December 2020

Published: 21 April 2021

Citation:

Tebaldi C (2021) Self-Organized Criticality in Economic Fluctuations:

The Age of Maturity.

Front. Phys. 8:616408.

doi: 10.3389/fphy.2020.616408

\section{INTRODUCTION}

The interaction between economic and physical sciences is so rich that a new interdisciplinary field has emerged, econophysics. While its cultural appealing is undisputable, contributions that are considered relevant and well disciplined by both economists and physicists are rare. It is fair to say that the self-organized critical (SOC) paradigm for economic and financial fluctuations put forward in the seminal contributions [1-3] is one of them.

While many are sympathetic with the proposal that an SOC paradigm underlies financial and macroeconomic fluctuations, the introduction of a common language and of analytic tools useful to make this proposal effective at both the descriptive and normative level are still underway. In fact, the virtue and the sin of the early SOC proposal lie in the use of a simple toy model to exemplify a number of characteristics that are expected to play a paradigmatic role.

It is virtually impossible to produce a systematic discussion of the overall state of the art, given the relevance of the topic and the number of research contributions in this area. Hence, in this short review, I propose a guided tour through the research spillover originating from those seminal proposals and argue that the SOC paradigm is an important building block of an emerging interdisciplinary paradigm suitable for framing a notion of statistical equilibrium relevant for both social and natural sciences.

In writing this contribution, I will take a purely subjective point of view with the deliberate goal of highlighting not only the strengths but also the open issues that are to be clarified to make the notion of the SOC state a more disciplined and useful instrument of economic analysis.

The review is organized as follows: we will start focusing in the next Section $\mathbf{2}$ on those common misconceptions and cultural differences that have so far limited the interaction among researchers with such different backgrounds. Then, we dedicate a section to each one of the following three themes that we consider SOC- "identitarian." In Section 3, we revisit the original model trying to clarify why the notion of "spontaneous emergence of a critical state" fits well in the traditional economic debate about the origin of macroeconomic fluctuations. In Section 4, we review the 
interdisciplinary attempts to improve the econometric description of financial and economic fluctuations. In particular, we focus on those that analyze financial time series borrowing the conventional tools used to analyze critical phenomena in physics: scaling, universality, and renormalization. In Section 5, we review the growing body of literature focusing on the notion of "avalanche" that we interpret as a correlated sequence of spatiotemporal events. We conclude with a focus on those research directions that in our opinion should be targeted by the coming research efforts in order to complete our understanding of the SOC paradigm with the provision of effective policy instruments.

\section{ECONOMIC EQUILIBRIUM VERSUS STATISTICAL (NON)-EQUILIBRIUM}

While the use of probability and statistics is common in both natural and social sciences, its theoretical foundations are radically different [4]; the contribution written by L. Hansen on occasion of the attribution of the Nobel Prize for economic sciences offers a key insight that clarifies the critical challenges underlying the quantification of uncertainty in a dynamic stochastic economy. The line of attack to macrofinancial modeling proposed in Ref. [4] fits nicely within a nonequilibrium statistical mechanics analysis of open systems: "Exogenous shocks repeatedly perturb a dynamic equilibrium through the model's endogenous transmission mechanisms" [4] (page 946). As Hansen points out, macrofinancial modeling poses two challenges. The first one has a descriptive focus: to describe what is called "uncertainty outside the model," that is, to best reproduce statistical features and impulse response of measured real-world time series. This challenge fits well within a conventional statistical mechanical approach. The second challenge faced in economic modeling is that the model also describes inside uncertainty: “. . . agents inside our model, be it consumers, entrepreneurs, or policymakers, must also confront uncertainty as they make decisions. I refer to this as inside uncertainty ...." This second aspect is intimately related to the normative focus of economics: “The modeler's choice regarding insiders' perspectives on an uncertain future can have significant consequences for each model's equilibrium outcomes." It is this second objective that poses a formidable hurdle to the statistical mechanical description of economic fluctuations. This second requirement changes the description at the microlevel of the system qualitatively: description of individual economic behavior requires the introduction of a set of control variables to describe actions at the individual and collective levels. So the conventional mechanical description of a particle must be replaced by the micro-description of individual decision-making. While the emphasis in physics is more focused on the descriptive content, these considerations explain why economists often consider acceptable a poor description of outside uncertainty as an acceptable price to pay in order to achieve a better description of inside uncertainty that is necessary to account for the policy reaction of individuals.

At first sight, these fundamental issues look completely unrelated and far from the SOC paradigm. On the contrary, they have a strict connection. Intuitively, the SOC automata microscopic rules are those that describe the rational behavior of individuals. The choice of an individual depends crucially on the inference process that the decision-maker adopts to frame observed data in a model. The authors of Ref. [5] provide a thorough information theoretic analysis of the relationship between inference and emergence of critical behavior within an abstract statistical information theory framework. They show that inference procedures are likely to yield models which are close to singular values of parameters, akin to critical points in physics where phase transitions occur. Hence, following this argument, when inside uncertainty is taken into account, the emergence of critical behavior will look like the rule rather than the exception.

A similar, yet simpler example of a microfunded process is proposed in Ref. [6]. They model a game of strategic network formation where agents must collectively form a network in the face of the following trade-off: each agent receives benefits from the direct links it forms with others, but these links expose it to the risk of being hit by a cascading failure that might spread over multistep paths. They prove that the resulting optimal networks are, in a precise sense, situated just beyond a (percolation type) phase transition in the behavior of the cascading failures.

It is worth observing that in this economic detour, we touched statistical equilibrium physics in relation to not only the definition of a second-order critical point corresponding to the critical point for percolation but also the notion of a cascading failure that intrinsically requires the nonequilibrium dynamics. An economy is fundamentally an open system where the endogenous reaction is induced from the agents that reoptimize their decisions. This makes the overall response highly nonlinear and certainly not amenable to a standard equilibrium statistical physics treatment.

In light of these considerations, it is striking and puzzling that the SOC paradigm may often provide an effective and accurate description of many empirical stylized properties of a competitive (economic) equilibrium.

\section{SELF-ORGANIZATION AND THE "INVISIBLE HAND"}

During the early 1990s, many authors have emphasized the role of complexity and nonlinearity in economics and the potential interaction with the analysis of complex phenomena in natural sciences (for a review, see, e.g., [7]). It is within this framework that $[1,2]$ formulate the proposal that a SOC state may explain business cycle fluctuations in the level of economic activity indicators. In the original model, sharing many characteristics of the directed sandpile [8] and the stochastic failure sharing [9] models, critical fluctuations are driven by local interaction between customers and suppliers, forming a network of producers with non-convex technologies. One key original aspect of the SOC approach to the analysis of macrofinancial fluctuations is the role played by what is called the "largeeconomy limit" (see, e.g., [2]); “...we argue that aggregate 
fluctuations in production continue to occur in the largeeconomy limit ..."

From a statistical mechanical point of view, this statement may seem quite natural. It mimics the necessity to consider the socalled thermodynamic limit to match a model with the relevant observables. As discussed in the previous section, the extension of this argument to the analysis of macrofinancial fluctuations is far from trivial; it is the problem of aggregation that has to be analyzed more carefully. A more recent strand of research has highlighted the main role that the network of firm interaction plays in the propagation of shocks in production economies (see, e.g., $[10,11])$. Ref. [12] provides an extensive review and proposes a model calibrated on real data. An undisputable merit of these approaches is their compliance at both micro- and macro-levels with the basic economic principles that regulate the firm and consumer actions.

From the point of view of statistical mechanics, they are not completely satisfactory: a more disciplined approach to the analysis of the large-economy limit is proposed in Ref. [13], relying on random matrix theory. In this case, the authors show explicitly that a network economy triggered toward the critical point generates power-tail size distributions of firms. The authors conjecture that evolutionary and behavioral forces conspire to drive the economy toward marginal stability.

In a different economic context, the authors of Ref. [14] offer an important example, to our knowledge the first one, of a properly microfunded "snowball effect" that drives the spontaneous emergence of critical behavior in the largeeconomy limit; Ref. [14] presents a state-dependent pricing model that describes the inflation fluctuations driven by idiosyncratic shocks hitting the cost of price changes of individual firms. Firms' pricing behavior in equilibrium exhibits complementarity: the critical cascading effect is sparked by firms' repricing that reduces all the competitors' relative prices, thus inducing more of them to reprice. They model the cascade process relying on the classical theory of critical branching processes. Following a trend common to most of the mean field approaches to SOC models, see also [15].

\section{SOC AND ECONOMETRICS}

One of the strongest arguments in favor of the approach pursued by econophysics is the inadequacy of linear stationary models in providing a reliable description of the basic properties of macrofinancial time series models.

From an empirical point of view, a "minimalist" characterization of SOC models requires the spontaneous emergence of power-law distributions and scaling relationships (in particular, see, e.g., $[9,16]$ ); it postulates that the size of the response (the avalanche) to an exogenous idiosyncratic shock (the addition of a grain in the sandpile) follows a law satisfying a finite-size scaling ansatz:

$$
P_{s}(s, L):=s^{-\tau_{s}} F_{s}\left(\frac{s}{L^{D_{s}}}\right)
$$

Then, assuming that $F_{s}(x)$ has an exponential decay for $x \gg 1$, $L$ is a fundamental cutoff to the scale-invariant behavior.
Furthermore, the scale-invariant critical behavior also requires that the size of the response is related to its spatial extension $a$ and to its lifetime $T$ by homogeneous relations, that is, $s \sim a^{\gamma}$ and $s \sim T^{\beta}$. This implies that the finite-size scaling relation holds also for the distributions of $a$ and $T$ :

$$
\begin{aligned}
& P_{a}(a, L):=a^{-\tau_{a}} F_{a}\left(\frac{a}{L^{D_{a}}}\right), P_{T}(T, L):=T^{-\tau_{T}} F_{T}\left(\frac{T}{L^{D_{T}}}\right) \\
& \frac{D_{s}}{D_{a}}=\frac{\tau_{s}-1}{\tau_{a}-1}=\gamma, \quad \frac{D_{s}}{D_{T}}=\frac{\tau_{s}-1}{\tau_{T}-1}=\beta .
\end{aligned}
$$

Scaling Invariance is hardwired in the parametric description of the impulse response and are difficult if not impossible to derive within the class of linear stationary models. Reference to the SOC paradigm appears in all the early contributions [17-20]. One important debatable question is whether, beyond power laws, macrofinancial fluctuations show consistency with the full articulated set of observable implications that characterize dynamic critical phenomena in physics.

In fact, a joint test of power-law behavior, finite-size scaling and universality within a properly defined renormalization group fixed point, is still missing. In the following, we review the interesting results that are suggestive of the possibility that an attracting fixed point may underlie the SOC state properties of financial time series.

\subsection{Universality}

The emergence of universal scaling relations in financial markets has a relatively long history in relation to return data. It is a relatively more recent acknowledgment in relation to the market microstructure behavior; Ref. [21] formulates the so-called market microstructure invariance hypothesis. It claims and verifies the existence of a universal invariant quantity $I$ representing the average cost of a single bet. It is expressed in dollars, independent of the asset, and constant over time. Dimensional analysis suggests a relation of the form

$$
\frac{P Q}{I}=f\left(\sigma_{\mathrm{d}}^{2} \frac{Q}{V}\right)
$$

where $p$ is the share price in dollars, $\sigma_{\mathrm{d}}^{2}$ denotes the square daily volatility, $V$ denotes the total daily amount traded with bets, and $Q$ denotes the average volume of an individual bet. Invoking the Modigliani-Miller capital structure irrelevance principle yields $f(x) \sim x^{-1 / 2}$, which implies up to a numerical factor that

$$
I=\frac{\sigma_{\mathrm{d}} P Q^{3 / 2}}{V^{1 / 2}}:=\frac{\mathcal{R}}{N^{3 / 2}}
$$

where $\mathcal{R}:=\sigma_{\mathrm{d}} P V$ measures the total dollar amount of risk traded per day (also referred to as total exchanged risk or trading activity), while $N:=V / Q$ represents the number of daily bets for a given contract; Ref. [22] proposes a reformulation of the hypothesis that improves the finite-size scaling collapse. A thorough discussion of the potential role played by the SOC state in explaining market microstructure liquidity dynamics is given in Ref. [23]. In classical critical phenomena, universality finds its explanation in the simple observation that macroscopic 
(aggregate) properties will depend only on the stochastic limiting properties of a model that are not removed by a progressive integration (averaging) of micro-fluctuations. The progressive averaging procedure is part of the so-called renormalization group transformation.

\subsection{Renormalization Group}

A natural bridge between critical phenomena and stochastic modeling of financial time series is offered by the probabilistic interpretation of the renormalization group transformation proposed in Ref. [24]. In this formulation, the RG theory is nothing else than a stochastic limit theory for possibly correlated random variables. Fixed points for the semigroup generated iterating the RG transformation represent the achievable limit distributions. Only properties that survive to the iteration and are relevant to define the fixed point are macroscopically observable; Ref. [25] includes an "econometrics -friendly" introduction to RG transformations in time series analysis. They formalize an extension of the (real space) RG transformation introducing an RG operator, the $\mathcal{R}$ operator in paper's notation, that acts on the classical space of stationary square integrable time series.

The most flexible operational approach to stochastic modeling of financial return time series relying on the renormalization group approach is originally proposed in Ref. [26] and systematically exposed in Ref. [27]. It is grounded on a "fine-graining" procedure obtained "inverting" the real space renormalization group (RG hereafter) flow in the space of return probability distributions. In this case, the starting point of the procedure is the fixed point equation that characterizes the scale-invariant distribution. Recovery of the observed ones occurs considering a "cascading procedure" that tracks backward the conventional RG coarse-graining procedure. The merits and challenges of this approach are extensively discussed in Ref. [27]. This approach seems to offer the best setup to frame the probabilistic analysis of (possibly selforganized) critical behavior in time series. The key ingredients that in our opinion are necessary to characterize a SOC ensemble (a set of counterfactuals) are essentially two: i) an endogenous propagation dynamics that preserves scale invariance of conditional moments and ii) a random rescaling factor designed to embody exogenous influences also. The fine-graining procedure has found application in a number of interesting declinations (see [28-30]) that witness the applicative content of the theory.

One crucial observation, noticed in Ref. [16] for the SOC abelian sandpile and explored also in relation to the fine graining by the authors in Ref. [27], is that statistical description of impulse-response functions may require a multiscaling framework, an extension of the conventional finite-size scaling framework. In this case, the probability distribution can be seen as a superposition of scaleinvariant clusters with heterogeneous fractal properties. Multifractal formalism requires that the scaling properties of $P_{s}(s, L)$ are described by the spectrum:

$$
f(\alpha):=\frac{\log \left(\int_{L^{\alpha}}^{+\infty} P_{s}(x, L) d x\right)}{\log (L)}, \alpha:=\frac{\log (s)}{\log (L)} \text { for } L \rightarrow+\infty .
$$

Then, in the limit $L \rightarrow+\infty$, asymptotic moment scaling functions are determined by:

$$
\left\langle s^{q}\right\rangle^{L \rightarrow+\infty} \approx L^{\sigma(q)}, \sigma(q):=\sup _{\alpha}[\alpha q+f(\alpha)] .
$$

These equations are consistent with a multiplicative decomposition of the random propagation effects and cast a direct connection with the analysis of financial markets building on the similarity of volatility fluctuations with energy dissipation cascades in turbulence. Early contributions by the authors of Refs. $[31,32]$ established a strong analogy between turbulent cascades and volatility clustering. A new generation of refined multiscale models, like, for example, the multifractal random walk model [33], is capable of matching empirical properties of observed time series volatility.

Beyond scale invariance, characterization of the SOC behavior relies also on property ii), that is, the existence of random correlated sequences of micro-events (an avalanche in the SOC dictionary) that generate a breakdown of time translation invariance. Statistical description of dynamic clustering within a scale-invariant model is analyzed in Ref. [34]. This class of models is able to reproduce the stylized behavior of volatility intermittent decay after a main financial shock. As observed in Ref. [35], it parallels the Omori law in geophysics for the seismic activity after an earthquake of exceptional magnitude.

\section{AVALANCHE DYNAMICS}

We dedicate the last concluding section to the paradigmatic role played by the concept of avalanche that is as intuitive as difficult to formalize within a macrofinancial model. Along this review, we encountered already many notions that are close friends of the notion of avalanche: the reorganization of the supply chain economy in a competitive equilibrium that is hit by a shock, the sequence of correlated orders that is determined by a single trade decision, and the random rescaling factor in the "fine-grained" model. Last but not least, recent macrofinancial research has shown that propagation of financial shocks across financial institutions interconnected by the web of financial claims plays a major role in the unfolding of financial crises (see, e.g., [36]). Consideration of the financial system as a SOC state has provided great insight into the economic collapse following the subprime crises of 2008 and the following sovereign credit crisis in Europe. The authors of Ref. [37] provide an extensive and interdisciplinary review.

Despite the large amount of interesting empirical evidence and modelization efforts, research has not yet reached a consensus on a precise, empirically testable definition of avalanche dynamics. Intuitively, it is meant to be a dynamic counterpart to the selfsimilar ensemble of clusters that characterizes fluctuations in an 
equilibrium system close to the critical point, where susceptibility diverges. Here is where the tension between the equilibrium and nonequilibrium description of avalanche statistics is more evident. Consider, for example, the "Ising model" of the SOC paradigm, the abelian sandpile. In this case, the steady-state model has an exact characterization in terms of the $q \rightarrow 0$ limit of the equilibrium Potts model, and critical properties can be derived from its conformal invariance (see [38]). Attempts to extend the mapping and characterize the dynamic (nonequilibrium) scaling properties of avalanche clusters have so far not been successful.

In conclusion, the previous considerations highlighted that the search of a satisfactory SOC model for macrofinancial fluctuations after thirty years is far from the end. Indeed, the SOC paradigm is still a promising avenue toward a truly effective

\section{REFERENCES}

1. Bak P, Chen K, Scheinkman J, and Woodford M. Aggregate fluctuations from independent sectoral shocks: self-organized criticality in a model of production and inventory dynamics. Res Econ (1993) 47(1):3-30. doi:10.1016/00355054(93)90023-V

2. Scheinkman JA, and Woodford M. Self-organized criticality and economic fluctuations. Am Econ Rev (1994) 84(2):417-21.

3. Bak P, Tang C, and Wiesenfeld K. Self-organized criticality: an explanation of the 1/f noise. Phys Rev Lett (1987) 59(4):381. doi:10. 1103/PhysRevLett.59.381

4. Hansen LP. Nobel lecture: uncertainty outside and inside economic models. J Political Econ (2014) 122(5):945-87. doi:10.1086/678456

5. Mastromatteo I, and Marsili M. On the criticality of inferred models. J Stat Mech: Theory Exp (2011) 10:P10012. doi:10.1088/1742-5468/2011/10/P10012

6. Blume L, Easley D, Kleinberg J, Kleinberg R, and Tardos É. Network formation in the presence of contagious risk. In: Proceedings of the 12th ACM conference on electronic commerce; 2011 Jun 5-9; San Jose, CA. New York, NY: ACM (2011). p. 1-10.

7. Anderson PW. The economy as an evolving complex system. Cleveland, $\mathrm{OH}$ : CRC Press (2018).

8. Dhar D, and Ramaswamy R. Exactly solved model of self-organized critical phenomena. Phys Rev Lett (1989) 63(16):1659. doi:10.1103/PhysRevLett.63. 1659

9. Manna S. Two-state model of self-organized criticality. J Phys A Math Theor (1991) 24(7):L363. doi:10.1088/0305-4470/24/7/009

10. Carvalho V, and Gabaix X. The great diversification and its undoing. Am Econ Rev (2013) 103(5):1697-727. doi:10.1257/aer.103.5.1697

11. Acemoglu D, Akcigit U, and Kerr W. Networks and the macroeconomy: an empirical exploration. NBER Macroecon Annu (2016) 30(1):273-335. doi:10. 1073/pnas.1613559113

12. Taschereau-Dumouchel M. Cascades and fluctuations in an economy with an endogenous production network (2019). Available from: https://papers. ssrn.com/sol3/papers.cfm?abstract_id=3115854 (Accessed February 1, 2021).

13. Moran J, and Bouchaud J-P. Will a large economy be stable (2019). Available from: https://papers.ssrn.com/sol3/papers.cfm?abstract_id=3324209 (Accessed February 1, 2021).

14. Nirei M, and Scheinkman J. Self-organization of inflation volatility. Tokyo, Japan: Institute for Monetary and Economic Studies (2019).

15. Zapperi S, Lauritsen KB, and Stanley HE. Self-organized branching processes: mean-field theory for avalanches. Phys Rev Lett (1995) 75:4071-4. doi:10.1103/ PhysRevLett.75.407110.1103/PhysRevLett.75.4071

16. Tebaldi C, De Menech M, and Stella AL. Multifractal scaling in the bak-tangwiesenfeld sandpile and edge events. Phys Rev Lett (1999) 83(19):3952. doi:10. 1103/PhysRevLett.83.3952 interdisciplinary paradigm to characterize critical statistical fluctuations arising in strategic equilibria of interacting rational individuals.

\section{AUTHOR CONTRIBUTIONS}

The author confirms being the sole contributor of this work and has approved it for publication.

\section{FUNDING}

This research gratefully acknowledges the support of the MIUR-PRIN Bando 2017-prot. 2017TA7TYC.

17. Mantegna RN, and Stanley HE. Introduction to econophysics: correlations and complexity in finance. Cambridge, United Kingdom: Cambridge University Press (1999).

18. Gabaix X, Gopikrishnan P, Plerou V, and Stanley HE. A theory of power-law distributions in financial market fluctuations. Nature (2003) 423(6937): 267-70. doi:10.1038/nature01624

19. Gabaix X. Power laws in economics and finance. Annu Rev Econ (2009) 1(1): 255-94. doi:10.1146/annurev.economics.050708.142940

20. Cont R, and Bouchaud J-P. Herd behavior and aggregate fluctuations in financial markets. Macroecon Dyn (1997) [arXiv preprint cond-mat/ 9712318].

21. Kyle AS, and Obizhaeva AA. Market microstructure invariance: empirical hypotheses. Econometrica (2016) 84(4):1345-404. doi:10.3982/ ECTA10486

22. Bucci F, Lillo F, Bouchaud J-P, and Benzaquen M. Are trading invariants really invariant? trading costs matter. Quant Finance (2020) 20:1059-68. doi:10. 1080/14697688.2020.1741667

23. Fosset A, Bouchaud J-P, and Benzaquen M. Endogenous liquidity crises. J Stat Mech Theory Exp (2020) 6:063401. doi:10.1088/1742-5468/ab7c64

24. Jona-Lasinio G. Renormalization group and probability theory. Phys Rep (2001) 352(4-6):439-58. doi:10.1016/S0370-1573(01)00042-4

25. Ortu F, Severino F, Tamoni A, and Tebaldi C. A persistence-based wold-type decomposition for stationary time series. Quant Econ (2020) 11(1):203-30. doi:10.3982/QE994

26. Baldovin F, and Stella AL. Scaling and efficiency determine the irreversible evolution of a market. Proc Natl Acad Sci USA (2007) 104(50):19741-4. doi:10. 1073/pnas.0706046104

27. Zamparo M, Baldovin F, Caraglio M, and Stella AL. Scaling symmetry, renormalization, and time series modeling: the case of financial assets dynamics. Phys Rev E (2013) 88(6):062808. doi:10.1103/PhysRevE.88. 062808

28. Andreoli A, Caravenna F, Dai Pra P, and Posta G. Scaling and multiscaling in financial series: a simple model. Adv Appl Probab (2012) 44(4):1018-51. doi:10.1239/aap/1354716588

29. Baldovin F, Caporin M, Caraglio M, Stella AL, and Zamparo M. Option pricing with non-Gaussian scaling and infinite-state switching volatility. J Econom (2015) 187(2):486-97. doi:10.1016/j.jeconom. 2015.02.033

30. Peirano PP, and Challet D. Baldovin-stella stochastic volatility process and wiener process mixtures. Eur Phys J B (2012) 85(8):276. doi:10.1140/epjb/ e2012-30134-y

31. Mandelbrot BB, Fisher AJ, and Calvet LE. A multifractal model of asset returns (1997). Available from: https://papers.ssrn.com/sol3/papers.cfm?abstract_ id=78588 (Accessed February 1, 2021).

32. Ghashghaie S, Breymann W, Peinke J, Talkner P, and Dodge Y. Turbulent cascades in foreign exchange markets. Nature (1996) 381(6585):767-70. doi: $10.1038 / 381767 \mathrm{a} 0$ 
33. Bacry E, Delour J, and Muzy J-F. Multifractal random walk. Phys Rev E (2001) 64(2):026103. doi:10.1103/PhysRevE.64.026103

34. Baldovin F, Camana F, Caraglio M, Stella AL, and Zamparo M. Aftershock prediction for high-frequency financial markets' dynamics In: Econophysics of systemic risk and network dynamics. Berlin, Germany: Springer (2013). p. 49-58.

35. Lillo F, and Mantegna RN. Power-law relaxation in a complex system: Omori law after a financial market crash. Phys Rev E (2003) 68(1):016119. doi:10. 1103/PhysRevE.68.016119

36. Haldane AG, and May RM. Systemic risk in banking ecosystems. Nature (2011) 469(7330):351-5. doi:10.1038/nature09659

37. Roukny T, Bersini H, Pirotte H, Caldarelli G, and Battiston S. Default cascades in complex networks: topology and systemic risk. Sci Rep (2013) 3:2759. doi:10. 1038/srep02759
38. Dhar D. Theoretical studies of self-organized criticality. Physica A (2006) 369(1):29-70. doi:10.1016/j.physa.2006.04.004

Conflict of Interest: The author declares that the research was conducted in the absence of any commercial or financial relationships that could be construed as a potential conflict of interest.

Copyright $(2021$ Tebaldi. This is an open-access article distributed under the terms of the Creative Commons Attribution License (CC BY). The use, distribution or reproduction in other forums is permitted, provided the original author(s) and the copyright owner(s) are credited and that the original publication in this journal is cited, in accordance with accepted academic practice. No use, distribution or reproduction is permitted which does not comply with these terms. 